Some local definability theory for holomorphic functions

Wilkie, A J

2008

MIMS EPrint: 2012.5

Manchester Institute for Mathematical Sciences

School of Mathematics

The University of Manchester

\footnotetext{
Reports available from: http://eprints.maths.manchester.ac.uk/

And by contacting: The MIMS Secretary

School of Mathematics

The University of Manchester

Manchester, M13 9PL, UK
} 


\title{
Some local definability theory for holomorphic functions
}

\author{
A.J. Wilkie \\ Mathematical Institute \\ University of Oxford \\ 24-29 St Giles \\ Oxford OX1 3LB \\ UK
}

October 24, 2006

\begin{abstract}
Let $\mathcal{F}$ be a collection of holomorphic functions and let $\mathbb{R}(P R(\mathcal{F}))$ denote the reduct of the structure $\mathbb{R}_{a n}$ to the ordered field operations together with the set of proper restrictions (see below) of the real and imaginary parts of all functions in $\mathcal{F}$. We ask the question: Which holomorphic functions are locally definable (ie have their real and imaginary parts locally definable) in the structure $\mathbb{R}(P R(\mathcal{F}))$ ? It is easy to see that the collection of all such functions is closed under composition, partial differentiation, implicit definability (via the Implicit Function Theorem in one dependent variable) and Schwarz Reflection. We conjecture that this exhausts the possibilities and we prove as much in the neighbourhood of generic points. More precisely, we show that these four operations determine the natural pregeometry associated with $\mathbb{R}(P R(\mathcal{F}))$-definable, holomorphic functions.
\end{abstract}

\section{Introduction}

In this paper a holomorphic function is always understood to have domain an open subset of $\mathbb{C}^{n}$ for some $n$. If $\Delta$ is an open box in $\mathbb{C}^{n}$ with rational 
data (ie $\Delta=D_{1} \times \cdots \times D_{n}$ for some open rectangles $D_{1}, \ldots, D_{n}$ in $\mathbb{C}$ with Gaussian rational corners) and $F$ is a holomorphic function whose domain contains the closure of $\Delta, \bar{\Delta} \subseteq \operatorname{dom}(F)$, then we say that $\Delta$ is suitable for $F$, or if a point $\mathbf{a} \in \Delta$ is given, suitable for $F$ around $\mathbf{a}$. The holomorphic function $F \mid \Delta$ is then called a proper restriction of $F$.

Let $\mathcal{F}$ be a collection of holomorphic functions. We assume that $\mathcal{F}$ contains all polynomials with Gaussian rational coefficients (in any number of variables). Denote by $P R(\mathcal{F})$ the set of all proper restrictions of all functions in $\mathcal{F}$. Let $\mathbb{R}(P R(\mathcal{F}))$ be the expansion of the ordered field of real numbers by (the graphs of) the functions in $\operatorname{PR}(\mathcal{F})$, where we identify $\mathbb{C}$ with $\mathbb{R}^{2}$ in the usual way. A holomorphic function $F$ is called locally definable from $\mathcal{F}$ if all its proper restrictions are definable in the structure $\mathbb{R}(P R(\mathcal{F}))$, where "definable" always means first-order definable without parameters unless otherwise stated. Clearly a holomorphic function $F$ is locally definable from $\mathcal{F}$ if and only if for all $\mathbf{w} \in \operatorname{dom}(F)$ there is some $\Delta$ suitable for $F$ around $\mathbf{w}$ such that $F \mid \Delta$ is definable in $\mathbb{R}(P R(\mathcal{F}))$.

\subsection{Problem}

Given $\mathcal{F}$, characterize the class of functions locally definable from $\mathcal{F}$ in terms of complex-analytically natural closure conditions.

I cannot claim to solve 1.1 completely here. However, I do give a complex analytic characterization of the pregeometry arising from local definability, and hence answer 1.1 in neighbourhoods of generic points of $\mathbb{C}^{n}$.

Let us first make some simple observations concerning 1.1 which follow from the fact that in the structure $\mathbb{R}(P R(\mathcal{F}))$ we may definably separate out the real and imaginary parts of complex functions and apply definable constructions coming from real algebra and analysis to them (such as $\epsilon-\delta$ methods).

\subsection{Differentiation}

If $F: U \rightarrow \mathbb{C}$ is locally definable from $\mathcal{F}$ (where $U \subseteq \mathbb{C}^{n}$ ) and $1 \leq i \leq n$, then so is the partial derivative $\frac{\partial F}{\partial z_{i}}: U \rightarrow \mathbb{C}$. 


\subsection{Schwarz Reflection}

If $F: U \rightarrow \mathbb{C}$ is locally definable from $\mathcal{F}$ then so is its Schwarz Reflection $F^{S R}: U^{\prime} \rightarrow \mathbb{C}$, where $U^{\prime}:=\{\overline{\mathbf{z}}: \mathbf{z} \in U\}$ (the bar here denotes co-ordinatewise complex conjugation) and where $F^{S R}(\mathbf{z}):=\overline{F(\overline{\mathbf{z}})}$ for $\mathbf{z} \in U^{\prime}$. (Note that Schwarz Reflection commutes with taking proper restrictions.)

Our results are most conveniently stated if we assume from the outset that $\mathcal{F}$ is closed under differentiation and under Schwarz Reflection (which, in view of 1.2 and 1.3 does not affect 1.1) and we fix such an $\mathcal{F}$ for the rest of this paper. Our conjectured answer to 1.1 is, roughly speaking, that a function is locally definable from $\mathcal{F}$ if and only if it can be obtained (locally) from $\mathcal{F}$ by finitely many applications of composition and extractions of implicitly defined functions:

\subsection{Definition}

Let $F: U \rightarrow \mathbb{C}, f: V \rightarrow \mathbb{C}$ be holomorphic functions where $U \subseteq \mathbb{C}^{n+1}$, and $V \subseteq \mathbb{C}^{n}$. Then we say that $f$ is implicitly defined from $F$ if for all $\mathbf{w} \in V$, $\langle\mathbf{w}, f(\mathbf{w})\rangle \in U$ and $F(\mathbf{w}, f(\mathbf{w}))=0 \neq \frac{\partial F}{\partial z_{n+1}}(\mathbf{w}, f(\mathbf{w}))$.

\subsection{Implicit Definability}

Notice that if, in $1.4, F$ is locally definable from $\mathcal{F}$, then so is $f$. For if $\mathbf{a} \in V$ then by the Implicit Function Theorem we may choose a sufficiently small $\Delta$, suitable for $f$ around $\mathbf{a}$, and a rectangle $D$ in $\mathbb{C}$ such that $\Delta \times D$ is suitable for $F$ around $\langle\mathbf{a}, f(\mathbf{a})\rangle$ and has the further property that for each $\mathbf{w} \in \Delta$ there is a unique $u \in D$ such that $F(\mathbf{w}, u)=0$. Since this $u$ is necessarily equal to $f(\mathbf{w})$ (for small enough $\Delta$ ) and since the function $F \mid(\Delta \times D)$ is definable in the structure $\mathbb{R}(P R(\mathcal{F}))$, it follows that the function $f \mid \Delta$ is too. (No parameters are needed because $\Delta$ and $D$ have rational data.)

\subsection{Composition}

I leave the reader to check that if $F: U \rightarrow \mathbb{C}$ (where $U \subseteq \mathbb{C}^{n}$ ) and $G_{i}: V_{i} \rightarrow \mathbb{C}$ (where $V_{i} \subseteq \mathbb{C}^{m}$ for $i=1, \ldots, n$ ) are locally definable from $\mathcal{F}$, then so is their composition $F \circ\left\langle G_{1}, \ldots, G_{n}\right\rangle: \bigcap_{i=1}^{n} V_{i} \cap\left\langle G_{1}, \ldots, G_{n}\right\rangle^{-1}[U] \rightarrow \mathbb{C}$. (This is 
not an immediate consequence of the fact that definable functions are closed under composition: one does need to invoke the continuity of the $G_{i}$ 's.)

\subsection{Definition}

We denote by $\tilde{\mathcal{F}}$ the smallest class of functions containing $\mathcal{F}$ and closed under both composition and implicit definability.

Thus we have seen (1.5 and 1.6) that every function in $\tilde{\mathcal{F}}$ is locally definable from $\mathcal{F}$.

\subsection{Conjecture}

A function $F$ is locally definable from $\mathcal{F}$ if and only if for all $\mathbf{a} \in \operatorname{dom}(F)$ there exists a function $G \in \tilde{\mathcal{F}}$ with $\mathbf{a} \in \operatorname{dom}(G)$, and some $\Delta$ suitable for both $F$ and $G$ around a , such that $F|\Delta=G| \Delta$.

In order to be able to state what I can actually prove, I require the following

\subsection{Definition}

Let $X$ be any subset of $\mathbb{C}$. Then $\tilde{D}(X)$ denotes the set of all complex numbers of the form $F(\mathbf{w})$ where $F \in \tilde{\mathcal{F}}$ and $\mathbf{w}$ is a tuple from $X$ such that $\mathbf{w} \in \operatorname{dom}(F)$. The set $L D(X)$ is defined similarly except that $F$ is allowed to be any function locally definable from $\mathcal{F}$.

It follows immediately from the comment preceeding 1.8 that $\tilde{D}(X) \subseteq$ $L D(X)$ for all $X \subseteq \mathbb{C}$. The main result of this paper is the following

\subsection{Theorem}

The operators $L D$ and $\tilde{D}$ are both pregeometries on $\mathbb{C}$ and are identical. Further, the conjecture holds in neighbourhoods of generic points. In other words, if $a_{1}, \ldots, a_{n}$ are independent complex numbers (for either of the pregeometries) and $F$ is a function locally definable from $\mathcal{F}$ with $\mathbf{a}=\left\langle a_{1}, \ldots, a_{n}\right\rangle \in \operatorname{dom}(F)$, then there exists a function $G \in \tilde{\mathcal{F}}$ with $\mathbf{a} \in \operatorname{dom}(G)$, and some $\Delta$ suitable for both $F$ and $G$ around $\mathbf{a}$, such that 
$F|\Delta=G| \Delta$

Presumably a (positive) solution to 1.8 would, in addition to 1.10, require some sort of resolution of singularities. However, for many model-theoretic purposes 1.10 is sufficient: non-generic points may be dealt with by a suitable inductive hypothesis.

The reader familiar with early work on o-minimal expansions of the real field may have noticed by now that $\mathbb{R}(P R(\mathcal{F}))$ is a structure to which Gabrielov's theorem on reducts of $\mathbb{R}_{a n}$ applies (see [2]), and hence is model complete (and o-minimal). It is not hard to deduce from this that the analogue of 1.10 for real analytic functions, in particular where we replace $\mathcal{F}$ by the set of all real and imaginary parts of functions in $\mathcal{F}$, holds. That is, the real and imaginary parts of a function locally definable from $\mathcal{F}$ are (generically) locally equal to functions obtained from the real and imaginary parts of functions in $\mathcal{F}$ by finitely many applications of composition and extraction of implicitly defined (real analytic) functions. But it is by no means clear how to deduce from this that the (complex) locally defined function itself has (generically) such a characterization in terms of the (complex) functions themselves in $\mathcal{F}$. For example, in the algebraic case (where $\mathcal{F}$ is just the set of all polynomials with Gaussian rational coefficients) this amounts to showing that if $F(\mathbf{z})=F(\mathbf{x}+\sqrt{-1} \mathbf{y})=u(\mathbf{x}, \mathbf{y})+\sqrt{-1} v(\mathbf{x}, \mathbf{y})$ is holomorphic for $\mathbf{z}$ in an open neighbourhood of a generic n-tuple $\mathbf{z}_{0}=\mathbf{x}_{0}+\sqrt{-1} \mathbf{y}_{0}$ (ie the co-ordinates of $\mathbf{z}_{0}$ are algebraically independent over $\mathbb{Q}$ ), and if $u$ and $v$ are definable functions in the ordered field of real numbers, then $F\left(\mathbf{z}_{0}\right)$ is algebraic over $\mathbb{Q}\left(\mathbf{z}_{0}\right)$ (and, indeed, that a polynomial relationship $P\left(\mathbf{z}_{0}, F\left(\mathbf{z}_{0}\right)\right)=0$ extends to an open neighbourhood of $\mathbf{z}_{0}$ ). Even this special case of 1.10 does not seem obvious to me. In fact, this case arose out of a misinterpretation of mine of a question of Hrushovski, namely whether quantifier elimination for the complex field could be "easily deduced" from the deeper fact of quantifier elimination for the real ordered field. Neither of us can remember exactly what the precise formulation was (though Hrushovski now guesses that it probably had something to do with decidability) but, at any rate, I was motivated to revisit elimination procedures for the real field with a view to investigating to what extent they "preserve the Cauchy-Riemann equations", and this is really the issue here. Indeed, I suspect that 1.10 could be deduced using the methods of van den Dries from the important and influential paper [1], but my main point in this note is to connect real and complex definability via another pre- 
geometry associated with derivations where the Cauchy-Riemann equations (and Schwarz Reflection) may be applied directly.

Another reason for looking at locally definable functions is that it might help in studying expansions of the complex field by certain entire functions, such as the exponential function, and thereby settling Zilber's conjecture: is every $\langle\mathbb{C},+, \cdot$ exp $\rangle$-definable (with parameters) subset of $\mathbb{C}$ either countable or co-countable? (See [5].) Here we would take $\mathcal{F}$ to be the collection of all polynomials in $z_{1}, z_{2}, \ldots, e^{z_{1}}, e^{z_{2}}, \ldots$ (with Gaussian rational coefficients)note that this $\mathcal{F}$ is closed under differentiation and Schwarz Reflection-and the hope would be, as was successful in the real case (see [4]), that one could study the unrestricted complex exponential function modulo its restrictions, and 1.10 gives the necessary control on the latter. One should also remark here that because $\mathbb{R}(P R(\mathcal{F}))$ is an o-minimal structure, one has available the extensive theory, developed by Peterzil and Starchenko, of complex analysis over such structures (see their survey [3]).

Of course, we cannot fruitfully expand the real field by the unrestricted complex exponential function (ie by the unrestricted real exponential and sine functions) because this results in a highly wild structure (equivalent to second-order number theory). The point of 1.10 is that using first-order real definability methods in the study of restricted holomorphic functions does not take us outside the realms of complex geometry.

The plan of the proof of 1.10 is as follows. In the next section I shall show that both $L D$ and $\tilde{D}$ are pregeometries. Then I shall introduce another pregeometry on $\mathbb{C}$, denoted $D D$, via the class of derivations on the field $\mathbb{C}$ that respect (ie satisfy the chain rule for) all the functions in $\mathcal{F}$. It is easy to show that $\tilde{D}$ and $D D$ are identical, this being the analogue of the classical fact that if $k \subseteq K$ are fields of characteristic zero and $a \in K$, then $a$ is algebraic over $k$ if and only if every derivation on $K$ that vanishes on $k$ also vanishes at $a$. In the fourth section I set up the bijection between the class of derivations on $\mathbb{C}$ respecting the functions in $\mathcal{F}$ and the class of (pairs of) derivations on $\mathbb{R}$ respecting their real and imaginary parts, this being where the Cauchy-Riemann equations and Schwarz Reflection are used. Next I observe that the above pregeometric notions have analogues in the real analytic case (still over the structure $\mathbb{R}(P R(\mathcal{F}))$ ) and then derive from Gabrielov's theorem that the corresponding version of 1.10 holds in this case. (This might be new and, indeed, holds in general for expansions of the real field to which Gabrielov's theorem applies. But it is a very easy consequence 
of model completeness and I am not sure whether it is a particularly useful one in this form, since it only holds for archimedean models.) Finally, the required complex result, 1.10 itself, can now be read off from the bijective correspondence between the real and complex derivations.

\section{The operators $L D$ and $\tilde{D}$ are pregeometries}

Let us show that local definablity satisfies the axioms for a pregeometry.

Firstly, it follows immediately from 1.6 that $L D(L D(X)) \subseteq L D(X)$ for all $X \subseteq \mathbb{C}$, so it only remains to prove the Steinitz Exchange Principle, all the other axioms being trivially satisfied.

So suppose that $X \subseteq \mathbb{C}, a, b \in \mathbb{C}$ and that $a \in L D(X \cup\{b\})$. Then there exists a function $F$, locally definable from $\mathcal{F}$, and a tuple $\mathbf{w}$ from $X$ with $\langle\mathbf{w}, b\rangle \in \operatorname{dom}(F)$ such that $F(\mathbf{w}, b)=a$. Say $\mathbf{w}$ is an $n$-tuple and suppose first that $\frac{\partial^{i} F}{\partial z_{n+1}^{i}}$ vanishes at the point $\langle\mathbf{w}, b\rangle$ for all $i \geq 1$. Then the function $z_{n+1} \mapsto F\left(\mathbf{w}, z_{n+1}\right)$ is (defined and) constant on an open neighbourhood of $\mathrm{b}$, with value a. Let $q$ be a Gaussian rational lying in this neighbourhood and define $G:\{\mathbf{z}:\langle\mathbf{z}, q\rangle \in \operatorname{dom}(F)\} \rightarrow \mathbb{C}: \mathbf{z} \mapsto F(\mathbf{z}, q)$. Then clearly $G$ is locally definable from $\mathcal{F}$ and $G(\mathbf{w})=a$. So $a \in L D(X)$ in this case.

For the remaining case, let $i \geq 1$ be minimal such that $\frac{\partial^{i} F}{\partial z_{n+1}^{i}}$ does not vanish at $\langle\mathbf{w}, b\rangle$. Then we may suppose that $i=1$, for if $i \geq 2$ then just replace $F$ by $F+\frac{\partial^{i-1} F}{\partial z_{n+1}^{i-1}}$ (which is permissable by 1.2 and 1.6). Now define $H\left(\mathbf{z}, z_{n+1}, z_{n+2}\right):=F\left(\mathbf{z}, z_{n+2}\right)-z_{n+1}\left(\right.$ for $\left\langle\mathbf{z}, z_{n+2}\right\rangle \in \operatorname{dom}(F)$ and $\left.z_{n+1} \in \mathbb{C}\right)$ so that $H$ is clearly locally definable from $\mathcal{F}$. Also $H(\mathbf{w}, a, b)=$ $0 \neq \frac{\partial H}{\partial z_{n+2}}(\mathbf{w}, a, b)$. It follows from the Implicit Function Theorem that there exists a holomorphic function $g$ such that $g(\mathbf{w}, a)=b$ and, for all $\left\langle\mathbf{z}, z_{n+1}\right\rangle \in \operatorname{dom}(g), H\left(\mathbf{z}, z_{n+1}, g\left(\mathbf{z}, z_{n+1}\right)\right)=0 \neq \frac{\partial H}{\partial z_{n+2}}\left(\mathbf{z}, z_{n+1}, g\left(\mathbf{z}, z_{n+1}\right)\right)$. Then $g$ is implicitly defined from $H$ and hence, by 1.5, locally definable from $\mathcal{F}$. Thus $b \in L D(X \cup\{a\})$ in this case, and the proof of the Exchange Principle is complete.

Now notice that the argument above almost goes through for the operator $\tilde{D}$. The only thing missing is the fact (used in the second case) that the collection of functions under consideration be closed under differentiation. 


\section{$2.1 \quad$ Lemma}

(i) Let $U$ be an open subset of $\mathbb{C} \backslash\{0\}$. Then the function $\iota: U \rightarrow \mathbb{C}: z_{1} \mapsto$ $z_{1}^{-1}$ lies in $\tilde{\mathcal{F}}$.

(ii) $\tilde{\mathcal{F}}$ is closed under differentiation.

\section{Proof}

For (i), one readily checks that the function $\iota$ is implicitly defined from the polynomial $z_{1} \cdot z_{2}-1$ and so lies in $\tilde{\mathcal{F}}$. (I remind the reader that $\mathcal{F}$, and hence $\tilde{\mathcal{F}}$, contains all polynomials with Gaussian rational coefficients.)

For (ii), let $\mathcal{S}$ denote the set of all functions $F \in \tilde{\mathcal{F}}$ such that for all $i$, $\frac{\partial F}{\partial z_{i}} \in \tilde{\mathcal{F}}$. Certainly $\mathcal{F} \subseteq \mathcal{S}$ by our original assumption on $\mathcal{F}$, so we only need to show that $\mathcal{S}$ is closed under implicit definability and composition.

For the former, suppose that $f, F$ are as in 1.4, that $F$ lies in $\mathcal{S}$ and that $1 \leq i \leq n$. By differentiating the identity in 1.4 with respect to $z_{i}$ we obtain, for $\mathbf{w} \in \operatorname{dom}(f)$ :

$$
\frac{\partial f}{\partial z_{i}}(\mathbf{w})=\frac{\partial F}{\partial z_{i}}(\mathbf{w}, f(\mathbf{w})) \cdot\left(\frac{\partial F}{\partial z_{n+1}}(\mathbf{w}, f(\mathbf{w}))\right)^{-1} .
$$

It now follows from (i) and the fact that $\tilde{\mathcal{F}}$ is closed under composition that $\frac{\partial f}{\partial z_{i}} \in \tilde{\mathcal{F}}$. Since this also holds trivially for $i>n$ we see that $f \in \mathcal{S}$.

Now suppose that, in the notation of 1.6 , the functions $F, G_{1}, \ldots, G_{n}$ all lie in $\mathcal{S}$. I leave the reader to apply the chain rule to the composite function $F \circ\left\langle G_{1}, \ldots, G_{n}\right\rangle$ (and invoke the closure of $\tilde{\mathcal{F}}$ under composition) to see that each of its first partial derivatives lies in $\tilde{\mathcal{F}}$, and hence that the composite function itself lies in $\mathcal{S}$, as required.

As remarked above, we have now established the following

\subsection{Theorem}

The operators $L D$ and $\tilde{D}$ are both pregeometries on $\mathbb{C}$.

The following observation, which will be used repeatedly, captures the spirit of these pregeometries. It's proof may be extracted easily from the proof of 2.2 . 


\subsection{Lemma}

Let $\mathbf{w}=\left\langle w_{1}, \ldots, w_{n}\right\rangle \in \mathbb{C}^{n}$. Then $w_{1}, \ldots, w_{n}$ are $\tilde{D}$-dependent (respectively, $L D$-dependent) if and only if there exists a function $F \in \tilde{\mathcal{F}}$ (respectively, a function $F$ locally definable from $\mathcal{F}$ ) such that $\mathbf{w} \in \operatorname{dom}(F)$ and $F(\mathbf{w})=0$, but such that $F$ does not vanish identically on any open neighbourhood of $\mathbf{w}$ contained in $\operatorname{dom}(F)$.

\section{Derivations}

Let $K=\mathbb{R}$ or $\mathbb{C}$. By a derivation on $K$ I shall simply mean a $\mathbb{Q}$-linear map from $K$ to $K$ which, in the case $K=\mathbb{C}$, is also $\mathbb{Q}(\sqrt{-1})$-linear.

Suppose that $F$ is a holomorphic function (if $K=\mathbb{C}$ ) or a real analytic function (if $K=\mathbb{R}$ ). (A real analytic function is assumed to have domain an open subset of $\mathbb{R}^{n}$, for some $\left.n\right)$. Let $\mathbf{a}=\left\langle a_{1}, \ldots, a_{n}\right\rangle$ be a point of $\operatorname{dom}(F)$. Then we say that a derivation $\delta: K \rightarrow K$ respects $F$ at the point $\mathbf{a}$ if $\delta(F(\mathbf{a}))=\sum_{i=1}^{n} \partial_{i} F(\mathbf{a}) \cdot \delta\left(a_{i}\right)$, where $\partial_{i} F$ denotes the partial derivative of $F$ with respect to the $i$ th (real or complex) variable of $F$. If $\delta$ respects $F$ at all points of its domain then we say that $\delta$ respects $F$. (Thus, a derivation in the usual sense is just a derivation in our sense that respects mutiplication.)

Let $\mathcal{C}$ be a set of functions as above. We denote by $\operatorname{Der}_{K}(\mathcal{C})$ the set of derivations that respect all $F \in \mathcal{C}$. It is clear that $\operatorname{Der}_{K}(\mathcal{C})$ is a $K$-vector space (under pointwise operations).

\subsection{Definition}

Let $\mathcal{C}$ be as above. For a finite subset $X \subseteq K$ we define

$$
D D_{K}^{\mathcal{C}}(X):=\left\{a \in K: \text { for all } \delta \in \operatorname{Der}_{K}(\mathcal{C}) \text {, if } \delta[X]=\{0\} \text {, then } \delta(a)=0\right\} .
$$

For an arbitrary subset $X \subseteq K$ we define

$$
D D_{K}^{\mathcal{C}}(X):=\left\{a \in K: a \in D D_{K}^{\mathcal{C}}\left(X^{\prime}\right) \text { for some finite } X^{\prime} \subseteq X\right\} .
$$

\subsection{Lemma}

For any $\mathcal{C}$, the operator $D D_{K}^{\mathcal{C}}$ is well defined (ie the two cases in 3.1 agree when $X$ is finite) and is a pregeometry on $\mathbb{C}$. 


\section{Proof}

It is trivial to check that $D D_{K}^{\mathcal{C}}$ is a well defined operator and that it satisfes all the axioms for a pregeometry apart from, possibly, the Exchange Principle. (The axiom of finite character is built into the definition.) To see that the Exchange Principle holds too, let $X \subseteq K, a, b \in K$ and suppose that $a \notin D D_{K}^{\mathcal{C}}(X)$ and that $b \notin D D_{K}^{\mathcal{C}}(X \cup\{a\})$. Let $X^{\prime}$ be an arbitrary finite subset of $X$ and choose $\delta_{1}, \delta_{2} \in \operatorname{Der}_{K}(\mathcal{C})$ such that $\delta_{1}\left[X^{\prime}\right]=\{0\}, \delta_{1}(a) \neq 0$, and $\delta_{2}\left[X^{\prime} \cup\{a\}\right]=\{0\}, \delta_{2}(b) \neq 0$. Let $\delta:=\delta_{2}(b) \cdot \delta_{1}-\delta_{1}(b) \cdot \delta_{2}$. Then $\delta$ lies in the $K$-vector space $D e r_{K}^{\mathcal{C}}$. Further, $\delta\left[X^{\prime} \cup\{b\}\right]=\{0\}$ and $\delta(a) \neq 0$. So $a \notin D D_{K}^{\mathcal{C}}\left(X^{\prime} \cup\{b\}\right)$, and since $X^{\prime}$ was an arbitrary finite subset of $X$, $a \notin D D_{K}^{\mathcal{C}}(X \cup\{b\})$ as required.

We now concentrate on the case $K=\mathbb{C}$, and we write $D D$ for $D D_{\mathbb{C}}^{\mathcal{F}}$. Our aim for the rest of this section is to show that $D D$ and $\tilde{D}$ are the same pregeometry on $\mathbb{C}$. So we first prove the following

\subsection{Lemma}

$\operatorname{Der}_{\mathbb{C}}(\tilde{\mathcal{F}})=\operatorname{Der}_{\mathbb{C}}(\mathcal{F})$.

\section{Proof}

Obviously $\operatorname{Der}_{\mathbb{C}}(\tilde{\mathcal{F}}) \subseteq \operatorname{Der}_{\mathbb{C}}(\mathcal{F})$, so suppose that $\delta \in \operatorname{Der}_{\mathbb{C}}(\mathcal{F})$. Then $\delta$ respects every function in $\mathcal{F}$ and we must show that this is preserved by implicit definability and by composition. So suppose that $f, F$ are as in 1.4 and that $\delta$ respects $F$. Let $\mathbf{a} \in \operatorname{dom}(f)$. Then it follows that $0=\delta(F(\mathbf{a}, f(\mathbf{a})))=\sum_{i=1}^{n} \frac{\partial F}{\partial z_{i}}(\mathbf{a}, f(\mathbf{a})) \cdot \delta\left(a_{i}\right)+\frac{\partial F}{\partial z_{n+1}}(\mathbf{a}, f(\mathbf{a})) \cdot \delta(f(\mathbf{a}))$.

However, by differentiating the identity in 1.4 and evaluating at the point $\mathbf{w}=\mathbf{a}$ we see that $\frac{\partial F}{\partial z_{i}}(\mathbf{a}, f(\mathbf{a}))=-\frac{\partial F}{\partial z_{n+1}}(\mathbf{a}, f(\mathbf{a})) \cdot \frac{\partial f}{\partial z_{i}}(\mathbf{a})$ for all $i=1, \ldots, n$. By substituting these equations into the equation above, and cancelling the non-zero term $\frac{\partial F}{\partial z_{n+1}}(\mathbf{a}, f(\mathbf{a}))$, we obtain $\delta(f(\mathbf{a}))=\sum_{i=1}^{n} \frac{\partial f}{\partial z_{i}}(\mathbf{a}) \cdot \delta\left(a_{i}\right)$. Since $\mathbf{a} \in \operatorname{dom}(f)$ was arbitrary, this shows that $\delta$ respects $f$, as required.

Now suppose that, in the notation of $1.6, \delta$ respects the functions $F, G_{1}, \ldots, G_{n}$. Let $\mathbf{b}=\left\langle b_{1}, \ldots, b_{m}\right\rangle \in \operatorname{dom}\left(F \circ\left\langle G_{1}, \ldots, G_{n}\right\rangle\right)$. Then $\left\langle G_{1}(\mathbf{b}), \ldots, G_{n}(\mathbf{b})\right\rangle \in$ $\operatorname{dom}(F)$ and $\delta\left(F\left(G_{1}(\mathbf{b}), \ldots, G_{n}(\mathbf{b})\right)\right)=\sum_{i=1}^{n} \frac{\partial F}{\partial z_{i}}\left(G_{1}(\mathbf{b}), \ldots, G_{n}(\mathbf{b})\right) \cdot \delta\left(G_{i}(\mathbf{b})\right)$. Also, $\mathbf{b} \in \operatorname{dom}\left(G_{i}\right)$ and $\delta\left(G_{i}(\mathbf{b})\right)=\sum_{j=1}^{m} \frac{\partial G_{i}}{\partial z_{j}} \cdot \delta\left(b_{j}\right)$ for each $i=1, \ldots n$. 
By combining these equations and using the chain rule, we see that $\delta$ also respects the function $F \circ\left\langle G_{1}, \ldots, G_{n}\right\rangle$ at an arbitrary point $\mathbf{b}$ of its domain and this completes the proof.

\subsection{Theorem}

The operators $D D$ and $\tilde{D}$ are identical.

Proof

Let $X \subseteq \mathbb{C}$.

Suppose first that $\mathbf{a}=\left\langle a_{1}, \ldots, a_{n}\right\rangle$ is a tuple from $X$ and that $F$ is a function in $\tilde{\mathcal{F}}$ with $\mathbf{a} \in \operatorname{dom}(F)$. Let $\delta$ be any derivation in $\operatorname{Der}_{\mathbb{C}}(\mathcal{F})$ satisfying $\delta\left[\left\{a_{1}, \ldots, a_{n}\right\}\right]=\{0\}$. By 3.3, $\delta$ respects $F$ and it follows immediately that $\delta(F(\mathbf{a}))=0$. This shows that $\tilde{D}(X) \subseteq D D(X)$.

Now suppose that $w$ is any complex number such that $w \notin \tilde{D}(X)$.

By 2.2 and the general theory of pregeometries, we may choose a $\tilde{D}$ basis for $\mathbb{C}$ of the form $B \cup\{w\}$, where $w \notin B$ and $X \subseteq \tilde{D}(B)$. We construct a derivation $\delta \in \operatorname{Der}_{\mathbb{C}}(\mathcal{F})$ such that $\delta(w)=1$ and $\delta[B]=\{0\}$ (so that $\delta[X]=\{0\}$ by the first part of this proof). This shows that $w \notin D D(X)$ and completes the proof of the theorem.

To construct $\delta$, let $c$ be an arbitrary complex number and, using the fact that $c \in \tilde{D}(B \cup\{w\})$, pick a tuple $\mathbf{a}=\left\langle a_{1}, \ldots, a_{n}\right\rangle$ from $B$ and a function $F \in \tilde{\mathcal{F}}$ such that $\langle\mathbf{a}, w\rangle \in \operatorname{dom}(F)$ and $F(\mathbf{a}, w)=c$. I claim that $\frac{\partial F}{\partial z_{n+1}}(\mathbf{a}, w)$ depends only on $c$. For if also $G(\mathbf{a}, w)=c$ with $G \in \tilde{\mathcal{F}}$ and $\langle\mathbf{a}, w\rangle \in \operatorname{dom}(G)$ (and we may suppose that the $n$ and a are the same as before by adding vacuous variables to $F$ and $G$ ) then the function $F-G$ lies in $\tilde{\mathcal{F}}$ and vanishes at the $\tilde{D}$-generic $(n+1)$-tuple $\langle\mathbf{a}, w\rangle$. But then by 2.3, it vanishes on some open neighbourhood of $\langle\mathbf{a}, w\rangle$. Hence so do its partial derivatives, and the claim follows. Thus we may set $\delta(c):=\frac{\partial F}{\partial z_{n+1}}(\mathbf{a}, w)$. It is clear that $\delta$ is $\mathbb{Q}(\sqrt{-1})$-linear, and by taking $F$ to be the first and second projection function on $\mathbb{C}^{2}$ we see that $\delta[B]=\{0\}$ and $\delta(w)=1$ respectively. Finally, the fact that $\delta$ respects all functions in $\mathcal{F}$ follows from (indeed, it is an instance of) the chain rule, and I leave the easy details to the reader.

\section{Real versus complex derivations}

Let $\mathcal{C}$ be any collection of holomorphic functions. For each $n$-ary function $F \in \mathcal{C}$ there are two real valued, real analytic functions - the real and imag- 
inary parts of $F$ - with domain the open subset of $\mathbb{R}^{2 n}$ corresponding to $\operatorname{dom}(F)$ under the usual identification of $\mathbb{C}$ with $\mathbb{R}^{2}$. Let us denote by $\mathcal{C}_{\text {real }}$ the collection of all the real functions obtained in this way. Our aim in this section is to investigate the relationship between the $\mathbb{R}$-vector space $\operatorname{Der}_{\mathbb{R}}\left(\mathcal{C}_{\text {real }}\right)$ of derivations on $\mathbb{R}$ respecting all the functions in $\mathcal{C}_{\text {real }}$ and the $\mathbb{C}$-vector space $\operatorname{Der}_{\mathbb{C}}(\mathcal{C})$ of derivations on $\mathbb{C}$ respecting all the functions in $\mathcal{C}$.

We shall use the following convention. If $F: U \rightarrow \mathbb{C}$ is a holomorphic function, where $U \subseteq \mathbb{C}^{n}$, then the real and imaginary parts, $u, v$ say, of $F$ are the real analytic functions with domain $U_{\text {real }}:=\{\langle\mathbf{x}, \mathbf{y}\rangle=$ $\left.\left\langle\left\langle x_{1}, \ldots, x_{n}\right\rangle,\left\langle y_{1}, \ldots, y_{n}\right\rangle\right\rangle \in \mathbb{R}^{2 n}: \mathbf{x}+\sqrt{-1} \mathbf{y} \in U\right\}$ satisfying $F(\mathbf{x}+\sqrt{-1} \mathbf{y})=$ $u(\mathbf{x}, \mathbf{y})+\sqrt{-1} v(\mathbf{x}, \mathbf{y})$ for $\langle\mathbf{x}, \mathbf{y}\rangle \in U_{\text {real }}$.

\subsection{Definition}

For $\lambda, \mu: \mathbb{R} \rightarrow \mathbb{R}$ any functions, the function $[\lambda: \mu]: \mathbb{C} \rightarrow \mathbb{C}$ is defined by $[\lambda: \mu](x+\sqrt{-1} y):=(\lambda(x)-\mu(y))+\sqrt{-1}(\lambda(y)+\mu(x))($ for $x, y \in \mathbb{R})$.

\subsection{Lemma}

If $\lambda$ and $\mu$ are derivations on $\mathbb{R}$ then $[\lambda: \mu]$ is a derivation on $\mathbb{C}$. Further, if $F$ is a holomorphic function with real and imaginary parts $u, v$, and domain $U \subseteq \mathbb{C}^{n}$, and if $\lambda, \mu$ both respect $u$ and $v$ at a point $\langle\mathbf{x}, \mathbf{y}\rangle \in U_{\text {real }}$, then $[\lambda: \mu]$ respects $F$ at the point $\mathbf{x}+\sqrt{-1} \mathbf{y}$.

\section{Proof}

Suppose that $\lambda$ and $\mu$ are derivations on $\mathbb{R}$. Then $[\lambda: \mu]$ is clearly $\mathbb{Q}$-linear. Further, for $x, y \in \mathbb{R}$,

$$
\begin{aligned}
{[\lambda: \mu](\sqrt{-1}(x+\sqrt{-1} y)) } & =[\lambda: \mu](-y+\sqrt{-1} x) \\
& =(-\lambda(y)-\mu(x))+\sqrt{-1}(\lambda(x)-\mu(y)) \\
& =\sqrt{-1}((\lambda(x)-\mu(y))+\sqrt{-1}(\lambda(y)+\mu(x))) \\
& =\sqrt{-1}[\lambda: \mu](x+\sqrt{-1} y)
\end{aligned}
$$

so $[\lambda: \mu]$ is also $\mathbb{Q}(\sqrt{-1})$-linear.

As for the second part, let us write $u_{i}$ for $\frac{\partial u}{\partial x_{i}}(\mathbf{x}, \mathbf{y})$ and $u_{n+i}$ for $\frac{\partial u}{\partial y_{i}}(\mathbf{x}, \mathbf{y})$ (for $i=1, \ldots, n$ ) and similarly for $v$. Then by 4.1 , the fact that $\lambda, \mu$ respect $u, v$ at $\langle\mathbf{x}, \mathbf{y}\rangle$, and the Cauchy-Riemann equations in the form $u_{n+i}=-v_{i}$, $v_{n+i}=u_{i}($ for $i=1, \ldots, n)$ we obtain 


$$
\begin{aligned}
{[\lambda: \mu](F(\mathbf{x}+\sqrt{-1} \mathbf{y}))=} & (\lambda(u(\mathbf{x}, \mathbf{y}))-\mu(v(\mathbf{x}, \mathbf{y})))+\sqrt{-1}(\lambda(v(\mathbf{x}, \mathbf{y}))+\mu(u(\mathbf{x}, \mathbf{y}))) \\
= & \sum_{i=1}^{n}\left[u_{i} \lambda\left(x_{i}\right)+u_{n+i} \lambda\left(y_{i}\right)-v_{i} \mu\left(x_{i}\right)-v_{n+i} \mu\left(y_{i}\right)\right. \\
& \left.+\sqrt{-1}\left(v_{i} \lambda\left(x_{i}\right)+v_{n+i} \lambda\left(y_{i}\right)+u_{i} \mu\left(x_{i}\right)+u_{n+i} \mu\left(y_{i}\right)\right)\right] . \\
= & \sum_{i=1}^{n}\left(u_{i}+\sqrt{-1} v_{i}\right)\left(\lambda\left(x_{i}\right)-\mu\left(y_{i}\right)+\sqrt{-1}\left(\lambda\left(y_{i}\right)+\mu\left(x_{i}\right)\right)\right) \\
= & \sum_{i=1}^{n} \frac{\partial F}{\partial z_{i}}(\mathbf{x}+\sqrt{-1} \mathbf{y}) \cdot\left([\lambda: \mu]\left(x_{i}+\sqrt{-1} y_{i}\right)\right) .
\end{aligned}
$$

Thus $[\lambda: \mu]$ respects $F$ at the point $\mathbf{x}+\sqrt{-1} \mathbf{y}$, as required.

Now suppose that $\delta$ is any derivation on $\mathbb{C}$. Then, in particular, there exist functions $\lambda, \mu: \mathbb{R} \rightarrow \mathbb{R}$ such that $\delta(x)=\lambda(x)+\sqrt{-1} \mu(x)$ for $x \in \mathbb{R}$. Clearly $\lambda$ and $\mu$ are $\mathbb{Q}$-linear, ie they are derivations on $\mathbb{R}$. Further, for all $x, y \in \mathbb{R}$,

$$
\begin{aligned}
{[\lambda: \mu](x+\sqrt{-1} y) } & =(\lambda(x)-\mu(y))+\sqrt{-1}(\lambda(y)+\mu(x)) \\
& =(\lambda(x)+\sqrt{-1} \mu(x))+\sqrt{-1}(\lambda(y)+\sqrt{-1} \mu(y)) \\
& =\delta(x)+\sqrt{-1} \delta(y) \\
& =\delta(x+\sqrt{-1} y) \quad(\text { since } \delta \text { is } \mathbb{Q}(\sqrt{-1}) \text {-linear }) .
\end{aligned}
$$

Thus $\delta=[\lambda: \mu]$. We now go on to prove the main result of this section.

\subsection{Theorem}

Let $\mathcal{C}$ be any collection of holomorphic functions closed under Schwarz reflection (see 1.3). Then the elements of $\operatorname{Der}_{\mathbb{C}}(\mathcal{C})$ are precisely the maps of the form $[\lambda: \mu]: \mathbb{C} \rightarrow \mathbb{C}$ for $\lambda, \mu \in \operatorname{Der}_{\mathbb{R}}\left(\mathcal{C}_{\text {real }}\right)$.

\section{Proof}

It follows from 4.2 that if $\lambda, \mu \in \operatorname{Der}_{\mathbb{R}}\left(\mathcal{C}_{\text {real }}\right)$ then $[\lambda: \mu] \in \operatorname{Der}_{\mathbb{C}}(\mathcal{C})$. So let $\delta \in \operatorname{Der}_{\mathbb{R}}\left(\mathcal{C}_{\text {real }}\right)$. We have observed above that $\delta=[\lambda: \mu]$ for some derivatives $\lambda, \mu$ on $\mathbb{R}$ and it remains to show that $\lambda$ and $\mu$ both respect $\mathcal{C}_{\text {real }}$. To this end, let $u, v$ be the real and imaginary parts of some function $F: U \rightarrow \mathbb{C}$ lying in $\mathcal{C}$. Let $\langle\mathbf{x}, \mathbf{y}\rangle \in U_{\text {real }}$ (see the convention immediately preceding 4.1) and, to ease the notation, temporarily write $u, u_{i}$ and $u_{n+i}$ for the complex numbers $u(\mathbf{x}, \mathbf{y}), \frac{\partial u}{\partial x_{i}}(\mathbf{x}, \mathbf{y})$, and $\frac{\partial u}{\partial y_{i}}(\mathbf{x}, \mathbf{y})$ respectively (for $i=1, \ldots, n)$ and similarly for $v$.

Then since $\delta$ respects $F$ we have

$$
\delta(F(\mathbf{x}+\sqrt{-1} \mathbf{y}))=\sum_{i=1}^{n} \frac{\partial F}{\partial z_{i}}(\mathbf{x}, \mathbf{y}) \cdot \delta\left(x_{i}+\sqrt{-1} y_{i}\right)
$$


But $\delta=[\lambda: \mu], F(\mathbf{x}+\sqrt{-1} \mathbf{y})=u+\sqrt{-1} v$ and $\frac{\partial F}{\partial z_{i}}(\mathbf{x}, \mathbf{y})=u_{i}+\sqrt{-1} v_{i}$ (for $i=1, \ldots n$ ), and hence it follows from 4.1 by equating real and imaginary parts in (1) that

$$
\lambda(u)-\mu(v)=\sum_{i=1}^{n} u_{i}\left(\lambda\left(x_{i}\right)-\mu\left(y_{i}\right)\right)-v_{i}\left(\lambda\left(y_{i}\right)+\mu\left(x_{i}\right)\right)
$$

and

$$
\lambda(v)+\mu(u)=\sum_{i=1}^{n} u_{i}\left(\lambda\left(y_{i}\right)-\mu\left(x_{i}\right)\right)+v_{i}\left(\lambda\left(x_{i}\right)-\mu\left(y_{i}\right)\right)
$$

Now consider the Schwarz Reflection $F^{S R}: U^{\prime} \rightarrow \mathbb{C}$ of $F$ (see 1.3). We have $\mathbf{x}-\sqrt{-1} \mathbf{y} \in U^{\prime}$ and $F^{S R}(\mathbf{x}-\sqrt{-1} \mathbf{y})=\overline{F(\mathbf{x}+\sqrt{-1} \mathbf{y})}=u-\sqrt{-1} v$. Further, $\frac{\partial F^{S R}}{\partial z_{i}}(\mathbf{x}-\sqrt{-1} \mathbf{y})=u_{i}-\sqrt{-1} v_{i}$ for $i=1, \ldots, n$. Also, by hypothesis, $F^{S R} \in \mathcal{C}$ and so $\delta$ respects $F^{S R}$ at the point $\mathbf{x}-\sqrt{-1} \mathbf{y}$. Hence, by applying the argument above with $F^{S R}$ in place of $F$ and $\mathbf{x}-\sqrt{-1} \mathbf{y}$ in place of $\mathbf{x}+\sqrt{-1} \mathbf{y}$ we obtain the equations

$$
\lambda(u)+\mu(v)=\sum_{i=1}^{n} u_{i}\left(\lambda\left(x_{i}\right)+\mu\left(y_{i}\right)\right)+v_{i}\left(-\lambda\left(y_{i}\right)+\mu\left(x_{i}\right)\right)
$$

and

$$
-\lambda(v)+\mu(u)=\sum_{i=1}^{n} u_{i}\left(-\lambda\left(y_{i}\right)-\mu\left(x_{i}\right)\right)-v_{i}\left(\lambda\left(x_{i}\right)+\mu\left(y_{i}\right)\right) .
$$

From (2), (4) and the Cauchy-Riemann equations in the form $v_{i}=-u_{n+i}$, $u_{i}=v_{n+i}$ (for $\left.i=1, \ldots, n\right)$ we obtain the equations

$$
\begin{aligned}
& \lambda(u)=\sum_{i=1}^{n} u_{i} \lambda\left(x_{i}\right)-v_{i} \lambda\left(y_{i}\right)=\sum_{i=1}^{n} u_{i} \lambda\left(x_{i}\right)+u_{n+i} \lambda\left(y_{i}\right), \text { and } \\
& \mu(v)=\sum_{i=1}^{n} u_{i} \mu\left(y_{i}\right)+v_{i} \mu\left(x_{i}\right)=\sum_{i=1}^{n} v_{n+i} \mu\left(y_{i}\right)+v_{i} \mu\left(x_{i}\right),
\end{aligned}
$$

which show that $\lambda$ respects the function $u$ at the point $\langle\mathbf{x}, \mathbf{y}\rangle$ and that $\mu$ respects the function $v$ at $\langle\mathbf{x}, \mathbf{y}\rangle$. The corresponding conclusions for $\lambda, v$ and $\mu, u$ follow similarly from (3) and (5).

\subsection{Remark}

If $\delta$ is a derivation on the field $\mathbb{C}$ in the usual sense, and $\delta=[\lambda: \mu]$ (which determines $\lambda$ and $\mu$ uniquely: just consider $\delta \mid \mathbb{R}$ ), then $\lambda, \mu$ are derivations on the field $\mathbb{R}$ in the usual sense. This follows either by direct calculation or from 4.5 by taking $\mathcal{C}=\{h\}$, where $h: \mathbb{C} \rightarrow \mathbb{C}: z \mapsto \frac{z^{2}}{2}$, and observing that real multiplication is the imaginary part of $h$.

\section{The proof of the main theorem}

We first observe that the results of the first three sections have versions for real analytic functions (defined on open subsets of $\mathbb{R}^{n}$, for various $n$ ). 
So let us fix a collection, $\mathcal{E}$ say, of such functions. We assume that $\mathcal{E}$ is closed under partial differentiation and that it contains all polynomials with rational coefficients. We let $P R(\mathcal{E})$ denote the collection of all functions $f \mid \Delta$, where $f \in \mathcal{E}$ and where $\Delta$ is suitable for $f$, ie it is a product of open intervals, with rational endpoints, such that $\bar{\Delta} \subseteq \operatorname{dom}(f)$. Then $\mathbb{R}(P R(\mathcal{E}))$ denotes the expansion of the ordered field of real numbers by all functions in $P R(\mathcal{E})$. The definition of the notion of a function being locally definable from $\mathcal{E}$, and of the closure of $\mathcal{E}$ under composition and implicit definablility, which we denote by $\tilde{\mathcal{E}}$, go through as before: just replace "holomorphic" everywhere by "real analytic". Similarly, one defines the operators $\tilde{E}(\cdot)$ and $L E D(\cdot)$, of closure under functions in $\tilde{\mathcal{E}}$ and under functions locally definable from $\mathcal{E}$ respectively, and proves that they are both pregeometries on $\mathbb{R}$ with $\tilde{E}(X) \subseteq L E D(X)$ for all $X \subseteq \mathbb{R}$. The analogue of 2.3 also holds.

We now establish the real version of 1.10.

\subsection{Lemma}

$\tilde{E}$ and $L E D$ are identical pregeometries on $\mathbb{R}$.

\section{Proof}

Let $X \subseteq \mathbb{R}$. We must show that $L E D(X) \subseteq \tilde{E}(X)$ and it is clearly sufficient to consider the case where $X=\left\{s_{1}, \ldots, s_{n}\right\}$ is finite and $\mathbf{s}:=$ $\left\langle s_{1}, \ldots, s_{n}\right\rangle$ is $\tilde{E}$-generic (ie $s_{1}, \ldots, s_{n}$ are $\tilde{E}$-independent real numbers).

We shall use Gabrielov's Theorem (see [2]) which tells us that any reduct of the structure $\mathbb{R}_{a n}$ in which the collection of basic functions of the language is closed under differentiation, has a model complete theory. This clearly applies to our structure $\mathbb{R}(P R(\mathcal{E}))$.

We set $k=\tilde{E}\left(\left\{s_{1}, \ldots, s_{n}\right\}\right)$ and observe that $k$ is a subfield of $\mathbb{R}$ (by the real version of $2.1(\mathrm{i})$ ) and is closed under all functions in $\tilde{\mathcal{E}}$. The proof of the lemma will be complete if we can show that the expansion of the field $k$ by the restriction to $k$ of (the graphs of) all functions in $P R(\mathcal{E})$ is existentially closed in $\mathbb{R}(P R(\mathcal{E}))$. For, by model completeness, this implies that this expansion is an elementary substructure of $\mathbb{R}(P R(\mathcal{E}))$ and hence closed under all (parameter-free) $\mathbb{R}(P R(\mathcal{E}))$-definable functions, whence $L E D\left(\left\{s_{1}, \ldots, s_{n}\right\}\right) \subseteq$ $k$, as required.

Now, by standard manipulations of existential formulas in languages expanding that of ordered fields, it is sufficient (in order to establish the required existential closedness) to prove the following 
Claim: Let $\mathbf{a} \in k^{r}, \mathbf{b} \in \mathbb{R}^{m}$ and $f \in \tilde{\mathcal{E}}$. Suppose further that $\Delta$ is suitable for $f,\langle\mathbf{a}, \mathbf{b}\rangle \in \Delta$ and $f(\mathbf{a}, \mathbf{b})=0$. Then there exists $\mathbf{b}^{\prime} \in k^{m}$ such that $\left\langle\mathbf{a}, \mathbf{b}^{\prime}\right\rangle \in \Delta$ and $f\left(\mathbf{a}, \mathbf{b}^{\prime}\right)=0$.

In fact, it is sufficient for our purposes to prove the claim just for functions $f$ lying in the compositional closure of $\mathcal{E}$, but, by stating the claim as we have, we may assume straight away that $r=n$ and that $\mathbf{a}=\mathbf{s}$. Now, to prove the claim, we pick a maximal $\tilde{E}$-independent subset of $\left\{b_{1}, \ldots, b_{m}\right\}$ over $\mathbf{s}$, where $\mathbf{b}=\left\langle b_{1}, \ldots, b_{m}\right\rangle$. Let us suppose, for notational convenience, that it is $\left\{b_{1}, \ldots, b_{l}\right\}$ (for some $\left.l=0, \ldots, m\right)$, so that $b_{l+1}, \ldots, b_{m} \in \tilde{E}\left(\left\{s_{1}, \ldots, s_{n}, b_{1}, \ldots, b_{l}\right\}\right)$. Say $\phi_{i}\left(\mathbf{s}, b_{1}, \ldots, b_{l}\right)=b_{l+i}$, where $\phi_{i} \in \tilde{\mathcal{E}}$, for $i=1, \ldots, m-l$. Define $g \in \tilde{\mathcal{E}}$ by $g\left(x_{1}, \ldots, x_{n+l}\right)=g(\mathbf{x}):=f\left(\mathbf{x}, \phi_{1}(\mathbf{x}), \ldots, \phi_{m-l}(\mathbf{x})\right)$, so that $g\left(\mathbf{s}, b_{1}, \ldots, b_{l}\right)=$ 0 . However, since $\left\langle\mathbf{s}, b_{1}, \ldots, b_{l}\right\rangle$ is an $\tilde{E}$-generic point of $\mathbb{R}^{n+l}$, it follows from the real version of 2.3 that $g$ vanishes on some open subset, $V$ say, of $\operatorname{dom}(g)$ with $\left\langle\mathbf{s}, b_{1}, \ldots, b_{l}\right\rangle \in V$. Thus we may pick rationals $q_{1}, \ldots, q_{l}$ sufficiently close to $b_{1}, \ldots, b_{l}$ (respectively) so that both $\left\langle\mathbf{s}, q_{1}, \ldots, q_{l}\right\rangle \in V$ and $\mathbf{b}^{\prime} \in \Delta$, where $\mathbf{b}^{\prime}$ denotes the $m$-tuple $\left\langle q_{1}, \ldots, q_{l}, \phi_{1}\left(\mathbf{s}, q_{1}, \ldots, q_{l}\right), \ldots, \phi_{m-l}\left(\mathbf{s}, q_{1}, \ldots, q_{l}\right)\right\rangle$. This choice of $\mathbf{b}^{\prime}$ clearly satisfies the conclusion of the claim, and hence the proof of the lemma is complete.

\subsection{Corollary}

Let $s_{1}, \ldots, s_{n}$ be $\tilde{E}$-independent real numbers and suppose that $g$ is a function locally definable from $\mathcal{E}$ with $\mathbf{s}:=\left\langle s_{1}, \ldots, s_{n}\right\rangle \in \operatorname{dom}(g)$. Then there exists a function $f \in \tilde{\mathcal{E}}$ with $\mathbf{s} \in \operatorname{dom}(f)$ such that $f=g$ on some open neighbourhood of $\mathbf{s}$.

\section{Proof}

Since, by definition, $g(\mathbf{s}) \in L E D\left(\left\{s_{1}, \ldots, s_{n}\right\}\right)$, it follows from 5.1 that there exists a function $f \in \tilde{\mathcal{E}}$ with $\mathbf{s} \in \operatorname{dom}(f)$ such that $f(\mathbf{s})=g(\mathbf{s})$. Then the function $f-g$ is locally definable from $\mathcal{E}$ and vanishes at the point $\mathbf{s}$. But $\mathbf{s}$ is also $L E D$-generic (by 5.1), so the result follows from the real version of 2.3 .

Suppose now that $\lambda$ is a derivation on $\mathbb{R}$ respecting every function in $\mathcal{E}$, ie $\lambda \in \operatorname{Der}_{\mathbb{R}}(\mathcal{E})$. The proof of 3.3 goes through with complex variables replaced by real (and no other changes), and so $\lambda \in \operatorname{Der}_{\mathbb{R}}(\tilde{\mathcal{E}})$. It now follows immediately from 5.2 that $\lambda$ respects every function locally definable from $\mathcal{E}$ at generic points of their domains. One has to work a little harder at 
non-generic points:-

\subsection{Lemma}

Let $\lambda \in \operatorname{Der}_{\mathbb{R}}(\mathcal{E})$. Then $\lambda$ respects every function locally definable from $\mathcal{E}$.

\section{Proof}

Let $f$ be a function locally definable from $\mathcal{E}$ and $\mathbf{s}=\left\langle s_{1}, \ldots, s_{n}\right\rangle$ a point of $\operatorname{dom}(f)$. Choose a maximal $\tilde{E}$-independent subset of $\left\{s_{1}, \ldots, s_{n}\right\}$ and suppose, for notational convenience, that it is $\left\{s_{1}, \ldots, s_{l}\right\}$, where $0 \leq l \leq n$. Now choose functions $\phi_{l+1}, \ldots, \phi_{n}$ in $\tilde{\mathcal{E}}$ such that $\phi_{i}\left(\mathbf{s}^{\prime}\right)=s_{i}$ for $i=l+$ $1, \ldots, n$, where $\mathbf{s}^{\prime}:=\left\langle s_{1}, \ldots, s_{l}\right\rangle$.

Define the function $g$ by $g\left(\mathbf{x}^{\prime}\right)=f\left(\mathbf{x}^{\prime}, \phi_{l+1}\left(\mathbf{x}^{\prime}\right), \ldots, \phi_{n}\left(\mathbf{x}^{\prime}\right)\right)$, so that $g$ is locally definable from $\mathcal{E}$ and $g\left(\mathbf{s}^{\prime}\right)=f(\mathbf{s})$. Now by the discussion before the statement of the lemma, $\lambda$ respects the $\phi_{i}$ 's and $g$ at the point $\mathbf{s}^{\prime}$, so we have

$$
\lambda\left(s_{i}\right)=\lambda\left(\phi_{i}\left(\mathbf{s}^{\prime}\right)\right)=\sum_{j=1}^{l} \frac{\partial \phi_{i}}{\partial x_{j}}\left(\mathbf{s}^{\prime}\right) \cdot \lambda\left(s_{j}\right), \text { for } i=l+1, \ldots, n,
$$

and

$$
\begin{aligned}
\lambda(f(\mathbf{s}))=\lambda\left(g\left(\mathbf{s}^{\prime}\right)\right) & =\sum_{j=1}^{l} \frac{\partial g}{\partial x_{j}}\left(\mathbf{s}^{\prime}\right) \cdot \lambda\left(s_{j}\right), \\
& =\sum_{j=1}^{l}\left(\frac{\partial f}{\partial x_{j}}(\mathbf{s})+\sum_{i=l+1}^{n}\left(\frac{\partial f}{\partial x_{i}}(\mathbf{s}) \cdot \frac{\partial \phi_{i}}{\partial x_{j}}\left(\mathbf{s}^{\prime}\right)\right)\right) \cdot \lambda\left(s_{j}\right),
\end{aligned}
$$

(by the chain rule)

$$
\begin{aligned}
& =\sum_{j=1}^{l}\left(\frac{\partial f}{\partial x_{j}}(\mathbf{s}) \cdot \lambda\left(s_{j}\right)+\sum_{i=l+1}^{n}\left(\frac{\partial f}{\partial x_{i}}(\mathbf{s}) \cdot\left(\sum_{j=1}^{l} \frac{\partial \phi_{i}}{\partial x_{j}}\left(\mathbf{s}^{\prime}\right) \cdot \lambda\left(s_{j}\right)\right),\right.\right. \\
& =\sum_{j=1}^{n}\left(\frac{\partial f}{\partial x_{j}}(\mathbf{s}) \cdot \lambda\left(s_{j}\right), \quad \text { by }(*) .\right.
\end{aligned}
$$

So $\lambda$ respects $f$ at $\mathbf{s}$, as required.

We can now prove that $L D$ and $\tilde{D}$ are identical pregeometries on $\mathbb{C}$.

Let $X \subseteq \mathbb{C}$. It remains to show that $L D(X) \subseteq \tilde{D}(X)$. So let $w \in L D(X)$ and choose a function $F$, locally definable from $\mathcal{F}$, and elements $a_{1}, \ldots, a_{n}$ of $X$ such that $\mathbf{a}:=\left\langle a_{1}, \ldots, a_{n}\right\rangle \in \operatorname{dom}(F)$ and $F(\mathbf{a})=w$. I shall show that $w \in D D(X)$, which suffices by 3.4. Indeed, I shall show that $w \in$ $D D\left(\left\{a_{1}, \ldots, a_{n}\right\}\right)$.

So let $\delta$ be an element of $\operatorname{Der}_{\mathbb{C}}(\mathcal{F})$ vanishing on the set $\left\{a_{1}, \ldots, a_{n}\right\}$. Then by 4.3 (with $\mathcal{C}=\mathcal{F}$ - recall our assumption on $\mathcal{F}$ stated just after 1.3 ) we may choose $\lambda, \mu \in \operatorname{Der}_{\mathbb{R}}\left(\mathcal{F}_{\text {real }}\right)$ such that $\delta=[\lambda: \mu]$. Now since $\mathcal{F}$ contains (complex) multiplication it follows that $\delta$ is a derivation on $\mathbb{C}$ in the usual sense and hence, by 4.4 , both $\lambda$ and $\mu$ are derivations on $\mathbb{R}$ in the usual sense. 
So if we define $\mathcal{E}$ to be the union of $\mathcal{F}_{\text {real }}$ with the set of all polynomials with rational coefficients, it follows that $\lambda, \mu \in \operatorname{Der}_{\mathbb{R}}(\mathcal{E})$.

Now the function $F$ is locally definable from $\mathcal{F}$. So its real and imaginary parts are certainly locally definable from $\mathcal{E}$, and hence, by 5.3 , are respected by both $\lambda$ and $\mu$. But then, by $4.2, \delta$ respects $F$ at every point of its domain, in particular at the point a. Since $\delta\left(a_{i}\right)=0$ for each $i=1, \ldots, n$ it follows that $\delta(F(\mathbf{a})=0$, ie $\delta(w)=0$, and we are done.

The second part of 1.10 now follows by the same argument used to deduce 5.2 from 5.1 .

\section{References}

[1] L van den Dries, On the elementary theory of restricted elementary functions, J. Symbolic Logic 53 (1988), 796-808.

[2] A Gabrielov, Complements of subanalytic sets and existential formulas for analytic functions, Inv. Math. 125 (1996), 1-12.

[3] Y Peterzil and S Starchenko, "Complex-like" analysis in o-minimal structures, Proceedings of the RAAG Summer School Lisbon 2003: O-minimal Structures, Eds M. Edmundo, D Richardson, A J Wilkie (2005), 77-103.

[4] A J Wilkie, Model completeness results for expansions of the ordered field of real numbers by restricted Pfaffian functions and the exponential function, J Amer. Math. Soc. 9, No. 4, (1996), 1051-1094.

[5] B Zilber, Generalized analytic sets, Translation from Algebra i Logika, Novosibirsk, 36(4) (1997), 361-380. 